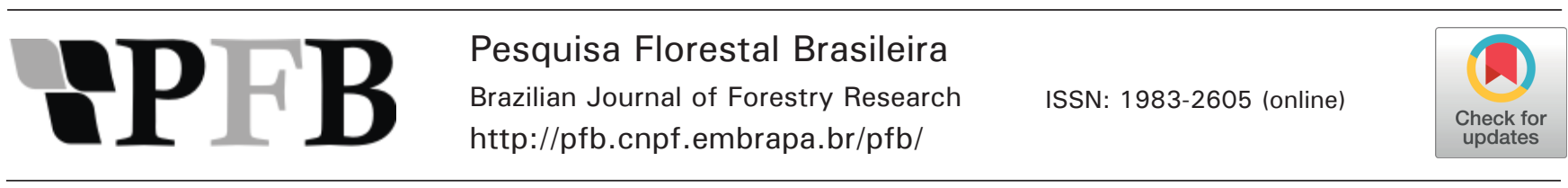

\title{
Modelagem do crescimento de Cryptomeria japonica por análise de tronco parcial
}

\author{
Vinícius Morais Coutinho1*, Ana Paula Dalla Corte ${ }^{1}$, Carlos Roberto Sanquetta ${ }^{1}$, Aurélio Lourenço Rodrigues ${ }^{1}$, \\ Mateus Niroh Inoue Sanquetta ${ }^{1}$
}

${ }^{1}$ Universidade Federal do Paraná, Av. Prof. Lothário Meissner 3400, Jardim Botânico, CEP 80210-170, Curitiba, PR, Brasil

\section{"Autor correspondente: \\ viniciusmorais@ufpr.br}

Termos para indexação:

Análise de probabilidades

Incremento

Manejo florestal

\section{Index terms:}

Probability analysis

Increment

Forest management

Histórico do artigo:

Recebido em 25/10/2016

Aprovado em 31/03/2017

Publicado em 30/06/2017

doi: 10.4336/2017.pfb.37.90.1371
Resumo - Este trabalho objetivou avaliar o padrão de crescimento bem com descrever a distribuição probabilística do incremento corrente anual em diâmetro de Cryptomeria japonica (L.F.) D. Don. em povoamento instalado no município de Rio Negro, PR. Foram amostradas 20 árvores em um plantio com 34 anos de idade e espaçamento $3 \mathrm{~m}$ x 2 m. Foram coletados discos a 1,3 m do solo (DAP) para a realização da análise de tronco parcial de cada árvore. A série de crescimento em diâmetro sem casca obtida foi utilizada para gerar as curvas de crescimento acumulado médio para a variável DAP (cm), de incremento médio anual (IMA) e incremento corrente anual (ICA). A partir dos dados de ICA, avaliou-se a distribuição de frequência desses por meio de funções de densidade de probabilidade (fdp's). O ICA do DAP foi de $0,78 \mathrm{~cm} \cdot \mathrm{ano}^{-1}$ e a idade de encontro das curvas de ICA e IMA ocorreu entre o $7^{\circ}$ e o $8^{\circ}$ ano. Identificou-se que cerca de $43 \%$ dos incrementos da espécie concentram-se até $0,5 \mathrm{~cm}$. Os resultados obtidos são úteis para a definição de estratégias para o manejo da espécie em regiões semelhantes ao local de estudo, definindo-se, por exemplo, idades de intervenção silvicultural, como o desbaste.

\section{Growth modeling of Cryptomeria japonica by partial trunk analysis}

\begin{abstract}
This study aimed to evaluate the growth pattern of Cryptomeria japonica increment (L. F.) D. Don. and to describe the probability distribution in stands stablished at the municipality of Rio Negro, Paraná State. Twenty trees were sampled in a 34 years-old stand, with $3 \mathrm{~m} \times 2 \mathrm{~m}$ spacing. Wood disks were taken from each tree at $1.3 \mathrm{~m}$ above the ground (DBH) to perform partial stem analysis. Diameter growth series without bark were used to generate the average cumulative growth curves for $\mathrm{DBH}(\mathrm{cm})$, mean annual increment (MAI) and current annual increment (CAI). From the increment data, the frequency distribution was evaluated by means of probability density functions (pdfs). The mean annual increment for DBH was $0.78 \mathrm{~cm}$.year ${ }^{-1}$ and the age of intersection of CAI and MAI curves was between the $7^{\text {th }}$ and 8 th years. It was found that near $43 \%$ of the species increments are concentrated bellow $0.5 \mathrm{~cm}$. The results are useful to define appropriate management strategies for the species for sites similar to the studying regions, defining for example ages of silvicultural intervention, such as thinning.
\end{abstract}

\section{Introdução}

As florestas plantadas fornecem matéria prima para os diversos setores da cadeia produtiva florestal, sendo responsáveis pela menor pressão sobre as florestas nativas. A área total de árvores plantadas no Brasil totalizou 7,8 milhões de ha em 2015, representando um crescimento de $0,8 \%$ em relação a 2014. Esses abrangem 
principalmente plantios de eucaliptos, pinus, acácia, teca, seringueira, paricá, araucária, pópulus, dentre outras espécies (Indústria Brasileira de Árvores, 2016).

A criptoméria (Cryptomeria japonica (L.F.) D. Don.) é uma espécie florestal conífera, pertencente à família Taxodiaceae e originária de regiões temperadas do Japão e da China (Shimizu \& Maiochi, 2007). Segundo esses autores, em suas origens, essa espécie atinge $20 \mathrm{~m}$ a $30 \mathrm{~m}$ de altura, podendo chegar a até 50 $\mathrm{m}$ em sítios excepcionais. Quanto ao diâmetro a 1,30 $\mathrm{m}$ do solo (DAP) alcançado por esta espécie em seus locais de origem, Kumagai et al. (2005) encontraram DAP médio de $27,1 \mathrm{~cm}$ em plantações, aos 51 anos, no Município de Kyushu, Japão. Genet et al. (2008) encontraram DAP médio de $21,2 \mathrm{~cm}$ em plantações, aos 30 anos, no município de Chongshou, China. No Brasil, onde é conhecida também como cedro-japonês ou pinheiro-japonês, a criptoméria pode atingir um incremento médio anual de até $45 \mathrm{~m}^{3}$.ha- ${ }^{-1}$ ano em sítios adequados (Carpanezzi et al., 1988). Para Carvalho (2001), C. japonica é uma das espécies arbóreas alternativas para plantios florestais no Brasil. Santos et al. (2000) comentam que a espécie possui grande potencial para plantios nas regiões altas e frias do Brasil, destacando-se pelo seu rápido crescimento, boa qualidade da madeira e boa adaptação ao clima e solo do Sul do país. No entanto, a criptoméria não vem sendo utilizada comercialmente no Brasil, embora já existam alguns estudos sobre o plantio e análise da madeira, principalmente considerando seu uso para produção de papel e celulose (Carpanezzi et al., 1988).

No manejo florestal é indispensável que se tenha o conhecimento do crescimento da floresta, para que haja um planejamento criterioso da produção por meio da prescrição de regimes de manejo apropriados. Para prognosticar a dinâmica de um povoamento florestal, se faz necessário lançar mão de técnicas que utilizam modelos de crescimento e produção. Esses modelos representam, em síntese, a dinâmica de desenvolvimento de uma árvore ou de uma floresta (Vanclay, 1994).

O estudo do crescimento possibilita conhecer o potencial de produção florestal de um determinado sítio e, com isso, orientar a tomada de decisões sob vários aspectos para o atendimento da demanda do mercado (Hosokawa et al., 1998).

Este trabalho teve por objetivo avaliar o padrão de crescimento do DAP $(\mathrm{cm})$, bem como descrever a distribuição probabilística dos incrementos correntes anuais (ICAs) em diâmetro sem casca, utilizando funções de crescimento e de densidade de probabilidade, em um povoamento de Cryptomeria japonica, aos 34 anos de idade.

\section{Material e métodos}

Para o desenvolvimento deste trabalho foram utilizados 20 indivíduos de Cryptomeria japonica coletados de um povoamento com 34 anos de idade não desbastado, plantado em espaçamento $3 \mathrm{~m} \mathrm{x} 2 \mathrm{~m}$ (1.667 árvores ha $\left.{ }^{-1}\right)$, localizado na Fazenda Experimental da Universidade Federal do Paraná, Rio Negro, PR. O município está situado a $26^{\circ} 06^{\prime} \mathrm{S}$ e $49^{\circ} 48^{\prime} \mathrm{W}$, com altitude de $847 \mathrm{~m}$. Este experimento foi instalado entre fevereiro e abril de 1980, com sementes originárias das províncias japonesas de Miyagi, Toyama, Shimane, Akita e Nara. Também foram utilizadas sementes originárias de Camanducaia, $\mathrm{MG}$, com a finalidade inicial de se realizar teste de procedência. Os indivíduos foram selecionados de forma a cobrir toda a amplitude diamétrica do povoamento, que variou entre $14,7 \mathrm{~cm}$ e 43,0 cm de DAP e entre $15,8 \mathrm{~m}$ e $27,65 \mathrm{~m}$ de altura.

A altura total de cada árvore (ht) foi medida após a derrubada. Na ocasião, discos foram retirados a 1,3 $\mathrm{m}$ do solo (DAP) para a realização da análise de tronco parcial. O material coletado foi seco em estufa com renovação de ar à temperatura de $65^{\circ} \mathrm{C}$, por aproximadamente 20 dias, até atingirem peso constante. $\mathrm{Na}$ sequência, os discos foram lixados, para em seguida serem submetidos à medição dos anéis. A variável idade foi determinada por meio da contagem dos anéis de crescimento das árvores e dos registros históricos. A Tabela 1 apresenta a distribuição das 20 árvores amostradas por classe de DAP.

Tabela 1. Distribuição diamétrica das árvores amostradas e médias dendrométricas.

\begin{tabular}{cccc}
\hline $\begin{array}{c}\text { Classes de DAP } \\
(\mathbf{c m})\end{array}$ & Frequência & $\begin{array}{c}\text { DAP médio } \\
(\mathbf{c m})\end{array}$ & $\begin{array}{c}\text { ht média } \\
(\mathbf{m})\end{array}$ \\
\hline $14-19,99$ & 5 & 17,28 & 17,87 \\
$20,00-25,99$ & 4 & 24,00 & 22,53 \\
$26,00-31,99$ & 6 & 28,68 & 23,05 \\
$32,00-37,99$ & 3 & 33,63 & 26,12 \\
$\geq 38,00$ & 2 & 41,85 & 24,00 \\
\hline TOTAL & 20 & & \\
\hline
\end{tabular}




\section{Análise de tronco parcial}

A medição dos anéis foi realizada de acordo com a metodologia proposta por Barusso (1977). Com os resultados da análise de tronco, foram obtidos pares de valores de diâmetro sem casca com a respectiva idade. Estes valores permitiram ajustar modelos de produção ou crescimento acumulado e, consequentemente, também possibilitaram gerar curvas de crescimento em diâmetro. A partir dos dados de incremento em diâmetro, foram ajustados cinco modelos de produção (Tabela 2), utilizando-se planilha eletrônica.

Tabela 2. Modelos de produção ajustados.

\begin{tabular}{|c|c|}
\hline Autor & Modelo \\
\hline Weibull & $f(x)=\beta_{0} \cdot\left(1-e^{-2_{1} \cdot x^{\beta_{2}}}\right)$ \\
\hline Logística & $f(x)=\beta_{0} /\left(1+\beta_{1} \cdot e^{-{ }^{2}{ }_{2} \cdot x}\right)$ \\
\hline Gompertz & $f(x)=\beta_{0} \cdot e^{-2_{1} \cdot e^{-2} 2 \cdot x}$ \\
\hline Schumacher & $f(x)=\beta_{0} \cdot e^{-{ }^{2} \cdot \cdot\left(\frac{1}{x}\right)}$ \\
\hline Chapman-Richards & $f(x)=\beta_{0} \cdot\left(1-e^{-_{1}{ }_{1} \cdot \mathrm{X}}\right)^{\beta_{2}}$ \\
\hline
\end{tabular}

Em que: $\mathrm{f}(\mathrm{x})=$ diâmetro acumulado sem casca $(\mathrm{cm}) ; e=$ número de Euler $(2,71828 \ldots) ; x=$ idade $(\operatorname{anos}) ; \beta_{0}, \beta_{1}, \beta_{2}$ e $\beta_{3}=$ coeficientes de regressão.

A avaliação dos modelos foi realizada seguindo três critérios de avaliação de desempenho para modelos não lineares (Schneider et al., 2009). Foram empregados os coeficientes de determinação ajustado $\left(\mathrm{R}^{2}{ }_{\mathrm{aj}}\right)$, bem como, o erro padrão da estimativa $\left(\mathrm{S}_{\mathrm{yx}}\right)$ e erro padrão da estimativa percentual $\left(\mathrm{S}_{\mathrm{yx}} \%\right)$. Adicionalmente, foi aplicado o critério de informação de Akaike (AIC), como um teste de aderência para os modelos de regressão. Foi empregada também a análise gráfica dos resíduos, que é um indicador fundamental para a avaliação de ajustes, objetivando-se visualizar os desvios existentes entre os valores reais e os valores estimados.

\section{Ajuste de funções de densidade e probabilidade}

Foram testadas as seguintes funções de densidade e probabilidade (fdp's) para descrever a distribuição probabilística dos incrementos em DAP: BirnbaumSaunders, Gamma, Gaussiana inversa, Log-logística e Log-normal. Todas as funções foram ajustadas utilizando-se o software Statgraphics. Para a seleção da melhor fdp aplicou-se o teste de Kolgomorov-Smirnov, a 5\% de significância.

\section{Resultados}

\section{Análise do crescimento}

Os resultados obtidos após ajuste dos modelos de produção estão apresentados na Tabela 3. Nota-se que os modelos testados apresentaram estatísticas de precisão bastante semelhantes. No entanto, o modelo de crescimento de Schumacher foi o que apresentou o melhor desempenho, com maior coeficiente de determinação ajustado $(0,727)$, menor erro padrão da estimativa $(25,54 \%)$, menor valor para o critério de Akaike $(1.815,16)$, e melhor distribuição gráfica dos resíduos. A partir da aplicação desse modelo, foi possível obter a informação do crescimento acumulado médio para a variável DAP sem casca $(\mathrm{cm})$, bem como as informações de incremento médio anual (IMA) e incremento corrente anual (ICA) (Figura 1).

Tabela 3. Resultados dos ajustes dos modelos de crescimento em diâmetro para Cryptomeria japonica em Rio Negro, PR.

\begin{tabular}{cccccccc}
\hline Modelo & $\boldsymbol{\beta}_{\mathbf{0}}$ & $\boldsymbol{\beta}_{\mathbf{1}}$ & $\boldsymbol{\beta}_{\mathbf{2}}$ & $\mathbf{R}_{\text {aj. }}^{2}$ & $\mathbf{S}_{\mathbf{y x}}$ & $\mathbf{S}_{\mathbf{y x}}(\mathbf{\%})$ & AIC \\
\hline Weibull & 322,74100 & 0,01071 & 0,62933 & 0,724 & 4,59 & 25,66 & $1.821,87$ \\
Logística & 26,36500 & 5,68082 & 0,18904 & 0,709 & 4,71 & 26,36 & $1.854,07$ \\
Gompertz & 27,40720 & 2,34579 & 0,13116 & 0,721 & 4,61 & 25,80 & $1.828,37$ \\
Schumacher & $\mathbf{3 2 , 7 9 7 7 0}$ & $\mathbf{7 , 2 2 2 2 9}$ & & $\mathbf{0 , 7 3 3}$ & $\mathbf{4 , 5 1}$ & $\mathbf{2 5 , 2 3}$ & $\mathbf{1 . 8 1 5 , 1 6}$ \\
Chapman-Richards & 31,47280 & 0,02526 & 2,52616 & 0,727 & 4,57 & 25,54 & $1.816,85$ \\
\hline
\end{tabular}

Em que: $\beta_{0}, \beta_{1}$ e $\beta_{2}=$ coeficientes de regressão, $\mathrm{R}_{\text {aj. }}^{2}=$ coeficiente de determinação ajustado; $\mathrm{S}_{\mathrm{yx}}=$ erro padrão da estimativa; e $\mathrm{AIC}=$ critério de informação de Akaike. 
Ao agrupar todas as árvores e analisar seus incrementos, nota-se que a curva de produção apresenta forma sigmoidal, em que a primeira fase corresponde à idade juvenil, a segunda à idade madura e a terceira à idade senil (Figura 1a). Cada fase mantém um ritmo de crescimento característico da vida total da árvore e juntas formam a curva de produção. De acordo com o modelo ajustado, o valor assintótico do DAP da espécie para as condições avaliadas é de aproximadamente $33 \mathrm{~cm}$ aos 34 anos de idade. No modelo de crescimento de Schumacher é possível notar que o parâmetro $\beta_{0}(32,79)$ assume o valor assintótico para o DAP. Esta propriedade é verificada no trabalho de Schumacher (1939).

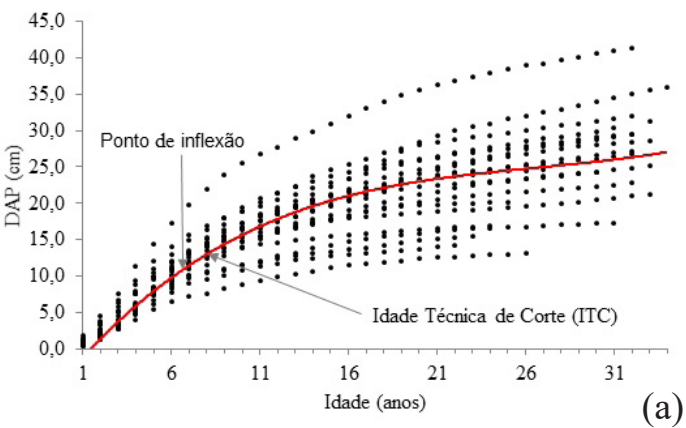

As curvas de ICA e IMA para a variável DAP se interceptaram próximas ao $8^{\circ}$ ano (Figura $1 \mathrm{~b}$ ), o que sugere esse momento como a idade ótima de desbaste, do ponto de vista técnico, pois com o envelhecimento do povoamento este fica mais susceptível a altas taxas de mortalidade dos indivíduos, a qual deve ser evitada por meio de desbastes. No oitavo ano, as árvores apresentaram em média 13,30 $\mathrm{cm}$ de DAP sem casca e incremento médio anual de $1,66 \mathrm{~cm}$. Aos 34 anos a floresta apresentou DAP médio de $26,52 \mathrm{~cm}$ com um incremento médio de $0,78 \mathrm{~cm}$ ano $^{-1}$.

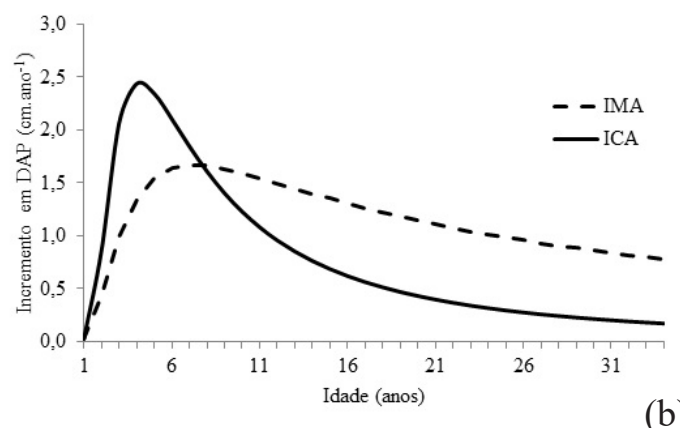

(b)

Figura 1. Curvas de crescimento acumulado para diâmetro a 1,30 m do solo (DAP) (a) e curvas de incremento médio anual (IMA) e incremento corrente anual (ICA) (b) para Cryptomeria japonica em Rio Negro, PR, obtidas por meio do modelo de Schumacher.

Observa-se na Figura 2 a evolução do incremento diamétrico médio ao longo da idade das árvores. Em termos absolutos, os valores que apresentaram maior variação ao longo do tempo foram os incrementos máximos, enquanto que os valores mínimos foram aqueles que apresentaram as menores variações. É possível observar que os incrementos médio, mínimo e máximo apresentam tendência decrescente após os oito anos de idade. Observou-se também que o maior incremento em DAP se deu próximo ao quinto ano.

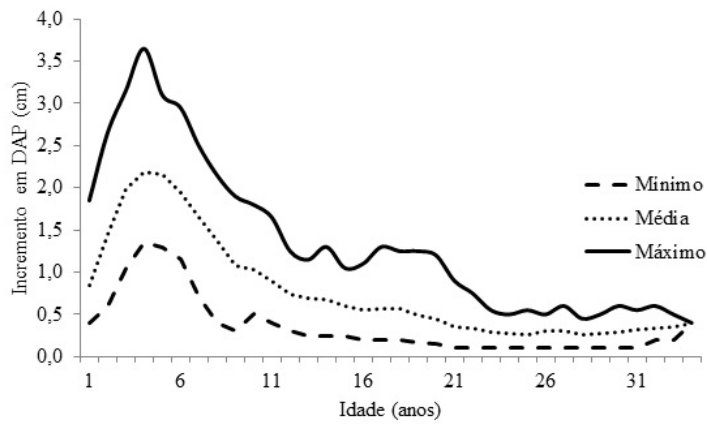

Figura 2. Valores de incremento mínimo, médio e máximo para o diâmetro a 1,30 $\mathrm{m}$ do solo (DAP) $(\mathrm{cm})$ para Cryptomeria japonica em Rio Negro, PR.

\section{Distribuição de frequência dos incrementos}

Na Tabela 4 está apresentado o teste de aderência Kolmogorov-Smirnov para as funções de densidade e probabilidade ajustadas para descrever a distribuição do ICA em diâmetro para Cryptomeria japonica. O teste de Kolgomorov-Smirnov revelou que dentre as funções testadas, apenas a de Birnbaum-Saunders e Gaussiana inversa foram satisfatórias para representar os incrementos, indicando aderência. Essa condição indica que as frequências observadas e esperadas não diferem estatisticamente entre si.

Tabela 4. Teste de Kolmogorov-Smirnov para as funções de densidade e probabilidade (fdp's) ajustadas para descrever a frequência do incremento em diâmetro para Cryptomeria japonica, aos 34 anos de idade.

\begin{tabular}{cc}
\hline Distribuição & Valor-p \\
\hline Birnbaum-Saunders & $0,130138^{\text {ns }}$ \\
Gamma & $0,000047^{*}$ \\
Gaussiana Inversa & $0,117877^{\text {ns }}$ \\
Log-logística & $0,027426^{*}$ \\
Log-normal & $0,036963^{*}$ \\
\hline
\end{tabular}

Em que: ns = não significativo ao nível de 5\% de significância, * = significativo ao nível de 5\% de significância. 
Foi selecionada a função de Birnbaum-Saunders, por ter apresentado a menor significância, ou seja, maior p-valor (Tabela 4). A função é dada pela equação 1.

$$
F(x)=\frac{1}{2 \sqrt{2 \pi \alpha \beta}}\left[\left(\frac{\beta}{x}\right)^{\frac{1}{2}}+\left(\frac{\beta}{x}\right)^{\frac{3}{2}}\right] e\left[-\frac{1}{2 \alpha^{2}}\left(\frac{x}{\beta}+\frac{\beta}{x}-2\right)\right]
$$

Em que: $\mathrm{x}=$ valores de incremento em diâmetro $(\mathrm{cm}), \alpha=$ 0,907466 (parâmetro alfa estimado), $\beta=0,589505$ (parâmetro beta estimado), $\mathrm{e}=$ número de Euler (2,71828...), $\pi=3,1416$.

Ao analisar os dados referentes às 597 observações, foi possível notar que os incrementos correntes anuais variaram entre $0,1 \mathrm{~cm}$ e $3,65 \mathrm{~cm}$. Observou-se também que a maioria das árvores não apresentou 34 anéis definidos, o que pode ser justificado pela não utilização de discos correspondentes à base da árvore. Destes, $42,79 \%$ correspondem a incrementos de até $0,5 \mathrm{~cm}$, $29,42 \%$ entre $0,51 \mathrm{~cm} \mathrm{e} 1,0 \mathrm{~cm}, 13,50 \%$ entre $1,01 \mathrm{~cm}$ e $1,5 \mathrm{~cm}, 6,7 \%$ entre $1,51 \mathrm{~cm}$ e $2,0 \mathrm{~cm}$ e $7,59 \%$ para incrementos maiores que $2,01 \mathrm{~cm}$ (Figura 3).

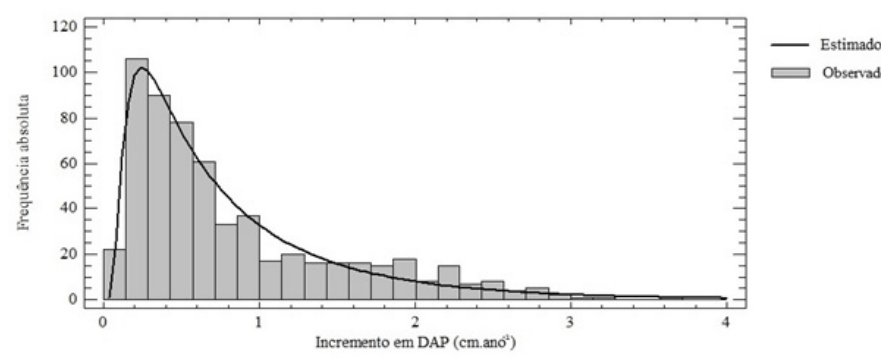

Figura 3. Valores observados e estimados pela função de Birnbaum-Saunders para o incremento corrente anual em diâmetro a 1,30 m do solo (DAP) para Cryptomeria japonica, em um povoamento com 34 anos de idade em Rio Negro, PR.

\section{Discussão}

A idade ótima para a realização do desbaste obtida no presente estudo se deu próximo aos 8 anos, onde há máxima produtividade da floresta para a variável diâmetro a 1,30 m do solo (DAP). Segundo Schneider (2008a), no manejo de povoamentos florestais, o conhecimento das inter-relações entre a densidade das árvores por área e o desenvolvimento em diâmetro são de extrema importância, pois com o envelhecimento do povoamento ocorre o aumento da taxa de mortalidade de indivíduos, a qual deve ser evitada por meio de intervenções silviculturais, como desbastes. No estudo de Dobner et al. (2013a), para Cryptomeria japonica em Rio Negro, PR, os autores observaram que a idade técnica de corte, em programas de manejo cujo objetivo fosse a máxima produção volumétrica no menor espaço de tempo, seria por volta dos 14 anos, momento em que houve o cruzamento das curvas de incremento médio anual (IMA) e incremento corrente anual (ICA) em diâmetro.

O crescimento das árvores não é regular ao longo da vida da árvore. Imaña-Encinas et al. (2005) mencionam que, para a maioria das espécies, observa-se uma diminuição do incremento em DAP ao longo dos anos. Para os indivíduos de criptoméria estudados notou-se tal diminuição. De modo geral, observa-se, portanto, que os maiores incrementos concentram-se na fase juvenil das árvores, onde naturalmente há maior vigor, quando comparado aos estágios de maturidade e senescência (Assmann, 1970; Husch et al., 1982). A existência de picos de incremento anual superior a $3,5 \mathrm{~cm}$ na fase juvenil da espécie indica bom potencial produtivo e adaptabilidade da espécie à região de estudo.

Neste trabalho foi possível observar que aproximadamente $43 \%$ dos valores de ICA observados para a espécie concentram-se até $0,5 \mathrm{~cm}$. A análise da distribuição de frequência dos incrementos é uma ferramenta útil, pois permite identificar classes de incremento passíveis de serem otimizadas. É possível aumentar a frequência em classes maiores de incremento por meio do manejo, ou seja, por meio da realização de desbastes, promovendo o incremento dos indivíduos remanescentes do povoamento (Schneider et al., 2008b). No entanto, Dobner et al. (2013b) citam que estudos silviculturais com povoamentos maiores em extensão, com desbastes e em diferentes classes de sítio, ainda são necessários para um maior entendimento do comportamento da espécie.

Destaca-se que, em razão do efeito da aceleração do crescimento descrito por Assmann (1970), a disponibilidade de recursos (e não o tempo) é o elemento mais importante na ontogenia e maturação dos indivíduos em um povoamento florestal. Desse modo, o aumento na disponibilidade de recursos por meio de desbastes na fase juvenil da espécie, ou ainda por meio da utilização de espaçamentos maiores, ao antecipar a culminação do incremento, antecipa invariavelmente a redução natural de sua taxa de crescimento (Pretzsch et al., 2015). Portanto, a produção comercial da espécie deve levar tais aspectos em consideração ao definir 
as estratégias de manejo mais adequadas, optando por antecipar ou retardar as idades de intervenção inicial ou rotação final, conforme os objetivos a que se destina a produção.

\section{Conclusão}

O modelo biológico de crescimento de Schumacher foi o que apresentou o melhor desempenho para descrever o crescimento em diâmetro de Cryptomeria japonica.

$\mathrm{O}$ encontro entre as curvas de IMA e ICA, do crescimento em diâmetro, configurou-se entre o $7^{\circ}$ e $8^{\circ}$ ano de plantio. Esse momento é sugerido como a idade ótima de desbaste, do ponto de vista técnico.

O povoamento estudado atingiu os maiores incrementos aos 5 anos, sendo que ao longo de 34 anos, $43 \%$ dos incrementos anuais concentraram-se até os 0,5 $\mathrm{cm}$. A função de densidade e probabilidade que melhor se ajustou aos dados e descreveu de maneira mais eficiente a distribuição dos incrementos correntes em diâmetro foi a função de Birnbaum-Saunders.

\section{Referências}

Assmann, E. The principles of forest yield study. New York: Pergamon Press, 1970. 506 p.

Barusso, A. P. Determinação de funções de crescimento mediante análise de tronco. 1977. 133 f. Dissertação (Mestrado em Engenharia Florestal) - Universidade Federal do Paraná, Curitiba. Não defendida.

Carpanezzi, A. A. et al. Zoneamento ecológico para plantios florestais no Estado de Santa Catarina. Curitiba: EMBRAPACNPF, 1988, 113 p. (EMBRAPA-CNPF. Documentos, 21).

Carvalho, P. E. R. Novas alternativas para reflorestamento. Revista da Madeira, v. 11, n. 60, p. 66-67, 2001.

Dobner Junior, M. et al. Aproveitamento do germoplasma de Cryptomeria japonica da estação experimental de Rio Negro, PR, visando a produção de sementes. Floresta, v. 43, n. 3, p. 363-372, 2013a. DOI: 10.5380/rf.v43i3.25050.

Dobner Junior, M. et al. Crescimento de um povoamento de Cryptomeria japonica no Sul do Brasil. Scientia Forestalis, v. 41, n. 97, p. $39-46,2013 b$.
Genet, M. et al. Root reinforcement in plantations of Cryptmeria japonica D. Don: effect of tree age and stand structure on slope stability. Forest Ecology and Management, v. 256, p. 1517-1526, 2008.

Hosokawa, R. T. et al. Introdução ao manejo e economia de florestas. Curitiba: Ed. da UFPR, 1998. 162 p.

Husch, B. et al. Forest mensuration. 3rd ed. New York: J. Willey \& Sons, 1982. 397 p.

Imaña-Encinas, J. et al. Idade e crescimento das árvores. Brasília, DF: UnB, 2005. 40 p. (UNB. Comunicações técnicas florestais, v. 7, n. 1).

Indústria Brasileira da Árvore. IBÁ: Indústria Brasileira da Árvore. Brasília, DF, 2016. 100 p. Relatório Ibá 2016.

Kumagai, T. et al. Sources of error in estimating stand transpiration using allometric relationships between stem diameter and sapwood área for Cyrptomeria japonica and Chamaecyparis obtusa. Forest Ecology and Management, v. 206, p. 191-195, 2005.

Pretzsch, H. et al. Ernest Assmann: a german pioneer in forest production ecology and quantitative silviculture. European Journal of Forest Research, v. 134, p. 391-402, 2015. DOI: 10.1007/ s10342-015-0872-4.

Santos, C. B. et al. Efeito do volume de tubetes e tipos de substratos na qualidade de mudas de Cryptomeria japonica (L. F.) D. Don. Ciência Florestal, v. 10, n. 2, p. 1-15, 2000. DOI: 10.5902/19805098.

Schneider, P. S. P. et al. Avaliação da relação densidade e diâmetro em povoamento de Pinus taeda. Ciência Florestal, v. 18, n. 4, p. 481-491, 2008a. DOI: 10.5902/19805098432.

Schneider, P. R. et al. Autodesbaste e diagrama de manejo da densidade em Povoamentos de Pinus taeda L. 2008b. 95 f. Dissertação (Mestrado em Engenharia Florestal) - Universidade Federal de Santa Maria, Santa Maria, RS.

Schneider, P. R. et al. Análise de regressão aplicada à engenharia florestal. Santa Maria, RS: UFSM, 2009. 294 p.

Shimizu, J. Y. \& Maiochi, R. A. Criptoméria como espécie alternativa para produção de madeira do Paraná. Pesquisa Florestal Brasileira, n. 54, p. 63-70, 2007.

Schumacher, F. X. A new growth curve and its application to timber yield studies. Journal Forestry, v. 37, p. 819-820, 1939.

Vanclay, J. K. Modelling forest growth and yield applications to mixed tropical forests. Walling Ford: CAB International, 1994. $312 \mathrm{p}$. 